\title{
COMMENTARY
}

\section{Emerging concepts in optimizing antimicrobial therapy of septic shock: speed is life but a hammer helps too}

\author{
Anand Kumar ${ }^{* 1}$ and Shravan Kethireddy² \\ See related research by Diaz-Martin et al., http://ccforum.com/content/16/6/R223
}

\begin{abstract}
Current research suggests that combination antimicrobial therapy where a given pathogen is covered by two or more antibiotics with differing antimicrobial mechanisms may be useful in a subset of critically ill patients, particularly those with septic shock. This paper elucidates the circumstances under which combination therapy may be useful by reinforcing the observation that beneficial effects are noted in critically ill patients with septic shock and, possibly, severe sepsis. Clinicians may wish to consider this approach with such patients.
\end{abstract}

The previous issue's article on combination therapy of severe sepsis and septic shock by Diaz-Martin and colleagues [1] opens the door to discussion regarding significant advances on this issue. For the most part, scientific research in this field over the past four decades has focused on the development of novel therapeutic targets [2]. Despite this effort, no therapies aimed at these novel targets are currently licensed. Current guidelines for management of sepsis provide guidance on the appropriate use of antimicrobials [3]; however, we have only recently begun to answer some fundamental questions of how to optimize antimicrobial therapy to maximize survival in sepsis and septic shock. For example, our study of a large international cohort of septic shock patients identified that the duration of hypotension prior to the administration of appropriate antimicrobials was a key determinant in survival [4]. The precipitous hourly decline in survival among patients inadequately treated

*Correspondence: akumar61@yahoo.com

'Sections of Critical Care Medicine and Infectious Diseases, University of Manitoba, Winnipeg, Manitoba, Canada R3A-1R9

Full list of author information is available at the end of the article with antimicrobials was startling. Despite this, only half of the patients in the cohort received appropriate antimicrobials in the first 6 hours following the onset of hypotension. These findings emphasize that earlier recognition and effective delivery of antimicrobial therapy improve survival in patients with septic shock. Other studies in recent years have examined the potential role of optimized pharmacokinetics of antimicrobial agents in sepsis and septic shock. Both those studies and our own are predicated on the idea that faster clearance of pathogens should result in improved outcomes in severe sepsis and septic shock.

This hypothesis raises the question of whether combined antimicrobial therapy could further improve survival in septic shock? In the previous issue of Critical Care, Diaz-Martin and colleagues [1] describe the effect of empiric combination antimicrobial therapy in patients with severe sepsis and septic shock. The results of this observational study, using a relatively large multicenter Spanish patient cohort, were impressive. Patients who received combination antimicrobial therapy experienced a $15 \%$ relative reduction in mortality and a $30 \%$ reduction in the odds of death when compared to patients who received single antibiotic therapy. The mechanisms responsible for this observed benefit, however, are difficult to identify. While a synergistic effect with enhanced clearance of pathogens is possible, the use of more than a single antimicrobial agent could also cast a broader net to capture potentially resistant infecting pathogens with higher frequency. Further, the results could be specific for a single national cohort and depend on the relative differences in broad antimicrobial resistance.

Our recent propensity matched analysis of several thousand patients with culture-positive septic shock suggested a similar benefit [5]. In that study, patients who received a combination of a $\beta$-lactam with either an aminoglycoside, fluoroquinolone or macrolide/clindamycin experienced greater hospital survival, as well as reduced days in the ICU and requirements for vasoactive and 
ventilator support when compared to patients who received monotherapy, survival benefits that extended across APACHE II (Acute Physiology and Chronic Health Evaluation II) severity-stratified subsets as well as among community and nosocomial infections [5]. We proposed that the basis for these effects was due to improved pathogen eradication (additive or synergistic effects) rather than increased breadth of coverage because each pathogen was know to be sensitive to at least one agent utilized.

Does this mean all patients with sepsis (as opposed to septic shock) should receive combination antimicrobial therapy? It appears that 'one size may not fit all'. We examined this question using pooled data from 62 datasets extracted from 50 published studies on antimicrobial therapy in severe bacterial infections [6]. While no overall benefit was found when we directly compared patients receiving combination versus monotherapy, we observed a dichotomous effect when patients were stratified by their risk of death. Among patients with a likelihood of death greater than $15 \%$, combination therapy reduced the odds of death by nearly $50 \%$ when compared to patients receiving monotherapy. The effect was particularly pronounced in patients with septic shock. On the other hand, a recent randomized multicenter trial in Germany comparing combination antimicrobial therapy with meropenam and moxifloxacin versus meropenam alone further complicates this issue [7]. No survival difference was seen between groups treated early for severe sepsis. How can we reconcile these disparate results?

We suggest the question is more complex than one of simple monotherapy versus combination therapy. The key issue may be the ability of combination therapy to augment bacterial clearance compared to monotherapy. If so, it is likely that augmented bacterial clearance with combination therapy may only be clinically relevant when the $\beta$-lactam component of combination therapy is less than maximally potent. The majority of pathogens causing septic shock are relatively sensitive to agents such as extended spectrum penicillins and first and second generation cephalosporins, which often achieve sub-maximal bacterial clearing with 60 to $70 \%$ time above the minimal inhibitory concentration $(\mathrm{T}>\mathrm{MIC})$ of the pathogen in the plasma. While this is adequate to improve survival for many serious infections, there is evidence to suggest that $100 \% \mathrm{~T}>\mathrm{MIC}$ may result in more effective clearance [8]. The use of high potency carbapenems will generate a time $>$ MIC of $100 \%$ and maximally rapid bacterial clearance for all but a few relatively resistant pathogens such as Pseudomonas. A second agent would not be expected to increase bacterial clearance (and correspondingly fail to improve outcome) in this circumstance.
Indeed, this insensitivity to combination therapy for regimens that included carbapenems was noted in our own analysis [5]. Beneficial effects of combination therapy were limited to regimens that utilized less than maximally potent antimicrobials, such as extended penicillins (for example, ampicillin, ticarcillin, pipercillin), semisynthetic penicillins (cloxacillin, oxacillin, and so on), as well as first, second and third generation (nonpseudomonal) cephalosporins. Agents with maximal potency in terms of $\mathrm{T}>\mathrm{MIC}$ for the majority of septic shock pathogens (carbapenems, ticarcillin/clavulanate, piperacillin/tazobactam, ceftazidime) yielded no benefit with the addition of a supplemental antibiotic.

Further study is clearly needed to address the question of under what circumstances combination therapy may be useful in sepsis and septic shock. Such a study should address shortcomings in the current extensive database of studies in this area. In particular, rather than asking the question of whether a specific antimicrobial combination is superior to a specific monotherapy in a specific infection within a relatively uniform microbial environment, a pragmatic study utilizing different commonly used antibiotic combinations in a variety of clinical conditions resulting in severe sepsis or septic shock would be most useful. In addition and perhaps most importantly, the specific logistics of the study should be developed on the basis of an underlying hypothesis of why combination antimicrobial therapy may be useful.

We suggest that the study should be designed based on the concept that enhanced bacterial clearance using synergistic drug combinations in septic shock will yield faster time to hemodynamic stability and improved survival. This suggests that the study would have to be done in an environment where the standard of therapy currently yields less than maximally rapid bacterial clearance (so that the addition of a supplemental agent could reasonably enhance bacterial clearance in a reasonable subset of cases). This could either be midlevel microbial resistance environments where agents such as high potency $\beta$-lactam/ $\beta$-lactamase inhibitor combinations and carbapenems for empiric therapy of severe sepsis/septic shock are not routinely utilized. Alternatively, the use of combination therapy with highly potent agents should, in theory, be of potential benefit in high microbial resistance environments. A prospective randomized study of this sort in combination with appropriate supplemental sub-studies of bacterial clearance would likely provide a definitive answer to the persistent question of the relative benefit of combination versus monotherapy in the critically ill patient with severe sepsis or septic shock.

Abbreviations

MIC, minimal inhibitory concentration. 


\section{Author details}

'Sections of Critical Care Medicine and Infectious Diseases, University of Manitoba, Winnipeg, Manitoba, Canada R3A-1 R9. ${ }^{2}$ Departments of Critical Care Medicine and Infectious Diseases, Geisinger Medical Center, Danville, PA 07822, USA.

Published: 15 January 2013

\section{References}

1. Diaz-Martin A, Martinez-Gonzalez ML, Ferrer R, Ortiz-Leyba C, Piacentini E, Lopez-Pueyo MJ, Martin-Loeches I, Levy MM, Artigas A, Garnacho-Montero J, the Edusepsis Study Group: Antibiotic prescription patterns in the empirical therapy of severe sepsis: combination of antimicrobials with different mechanisms of action reduce mortality. Crit Care 2012, 16:R223.

2. Suffredini AF, Munford RS: Novel therapies for septic shock over the past 4 decades. J Am Med Assoc 2011, 306:194-199.

3. Dellinger RP, Levy MM, Carlet JM, Bion J, Parker MM, Jaeschke R, Reinhart K, Angus DC, Brun-Buisson C, Beale R, Calandra T, Dhainaut JF, Gerlach H, Harvey M, Marini JJ, Marshall J, Ranieri M, Ramsay G, Sevransky J, Thompson BT, Townsend S, Vender JS, Zimmerman JL, Vincent JL: Surviving Sepsis Campaign: international guidelines for managment of severe sepsis and septic shock. Intensive Care Med 2008, 34:17-60.

4. Kumar A, Roberts D, Wood KE, Light B, Parrillo JE, Sharma S, Suppes R, Feinstein D, Zanotti S, Taiberg L, Gurka D, Kumar A, Cheang M: Duration of hypotension before initiation of effective antimicrobial therapy is the critical determinant of survival in human septic shock. Crit Care Med 2006, 34:1589-1596.
5. Kumar A, Zarychanski R, Light B, Parrillo J, Maki D, Simon D, Laporta D, Lapinsky S, Ellis P, Mirzanejad Y, Martinka G, Keenan S, Wood G, Arabi Y, Feinstein D, Kumar A, Dodek P, Kravetsky L, Doucette S; Cooperative Antimicrobial Therapy of Septic Shock (CATSS) Database Research Group: Early combination antibiotic therapy yields improved survival compare with monotherapy in septic shock: a propensity-matched analysis. Crit Care Med 2010, 38:1773-1785.

6. Kumar A, Safdar N, Kethireddy S, Chateau D: A survival benefit of combination antibiotic therapy for serious infections associated with sepsis and septic shock is contingent only on the risk of death: a metaanalytic/meta-regression study. Crit Care Med 2010, 38:1651-1664.

7. Brunkhorst FM, Oppert M, Marx G, Bloos F, Ludewig K, Putensen C, Nierhaus A, Jaschinski U, Meier-Hellmann A, Weyland A, Gründling M, Moerer O, Riessen R, Seibel A, Ragaller M, Büchler MW, John S, Bach F, Spies C, Reill L, Fritz H, Kiehntopf M, Kuhnt E, Bogatsch H, Engel C, Loeffler M, Kollef MH, Reinhart K, Welte T; German Study Group Competence Network Sepsis (SepNet): Effect of empirical treatment with moxifloxacin and meropenam vs meropenam on sepsis-related organ dysfunction in patients with severe sepsis. J Am Med Assoc 2012, 307:2390-2399.

8. Craig WA: Pharmacokinetic/pharmacodynamic parameters: Rationale for antibacterial dosing of mice and men. Clin Inf Dis 1998, 16:1-12.

doi:10.1186/cc11890

Cite this article as: Kumar A, Kethireddy S: Emerging concepts in optimizing antimicrobial therapy of septic shock: speed is life but a hammer helps too. Critical Care 2013, 17:104 